\title{
Anthropomorphic Soft Robotics - from Torque Control to Variable Intrinsic Compliance
}

\author{
A. Albu-Schäffer, O. Eiberger, M. Fuchs, M. Grebenstein, S. Haddadin, Ch. Ott, A. \\ Stemmer, T. Wimböck, S. Wolf, Ch. Borst and G. Hirzinger
}

\begin{abstract}
The paper gives an overview on the developments at the German Aerospace Center DLR towards anthropomorphic robots which not only try to approach the force and velocity performance of humans, but also have similar safety and robustness features based on a compliant behaviour. We achieve this compliance either by joint torque sensing and impedance control, or, in our newest systems, by compliant mechanisms (so called VIA - variable impedance actuators), whose intrinsic compliance can be adjusted by an additional actuator. Both approaches required highly integrated mechatronic design and advanced, nonlinear control and planning strategies, which are presented in this paper.
\end{abstract}

\section{Introduction}

Soft Robotics is an approach for designing and controlling robots which can interact with unknown environments and cooperate in a safe manner with humans, while approaching their performance in terms of weight, force, and velocity. These robots are expected to push forward not only such new application fields as medical robotics, robotized outer space and planetary exploration, or personal service and companion robotics, but also to drastically move the horizons of industrial automation. Today's industrial robots still operate in their huge majority in blind, position controlled mode, being dangerous to humans and thus having to be enclosed by protective fences. In contrast, this new generation of robots can share the space and the workload with the humans providing higher adaptability to product diversity and short production life cycles. However, it is clear that these human friendly robots will look very different from today's industrial robots. Rich sensory information, light-weight design and soft robotics features are required in order to reach the ex-

Institute of Robotics and Mechatronics

German Aerospace Center (DLR)

e-mail: alin.albu-schaeffer@dlr.de 

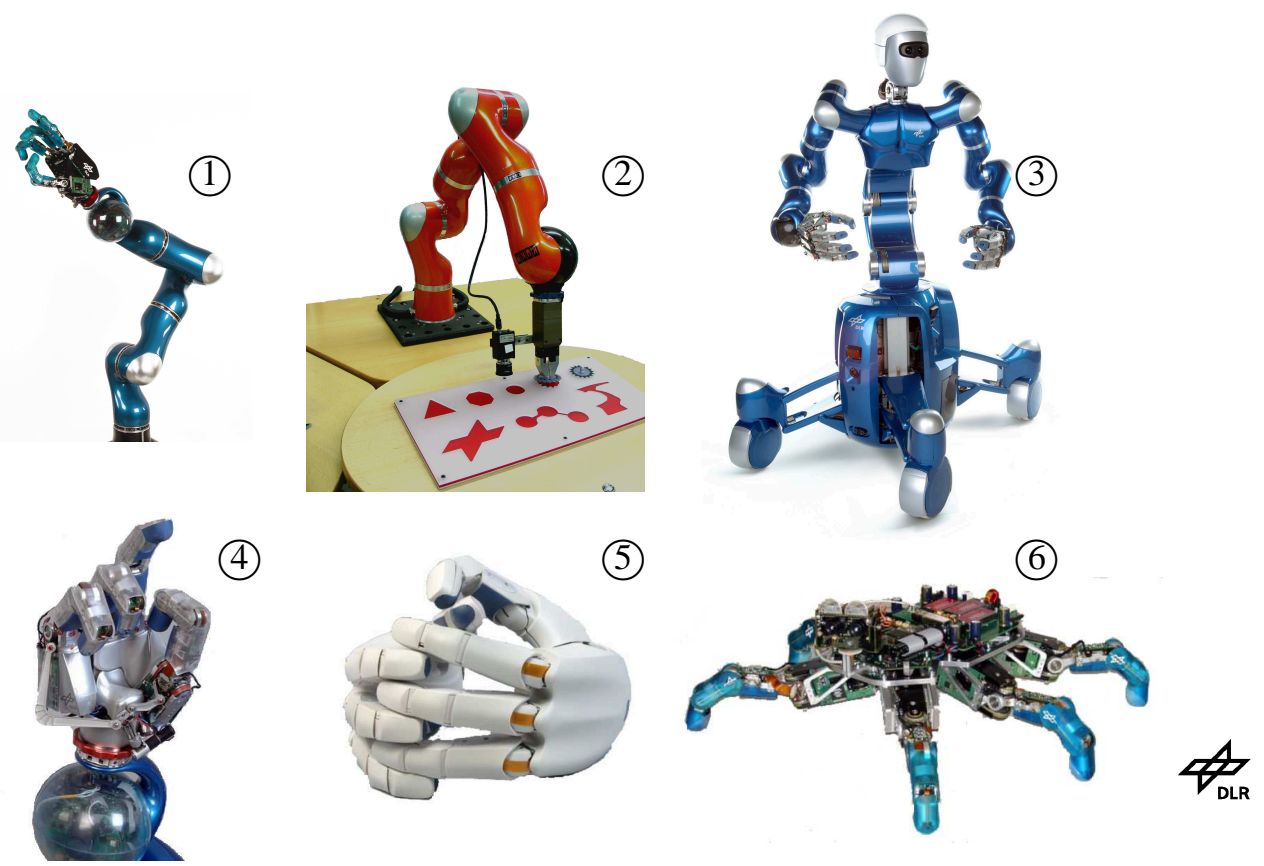

Fig. 1: Overview of the DLR Robots: (1): The DLR-LWRIII equipped with the DLR-HandII. (2): The DLR-KUKA-LWRIII which is based on the DLR-LWRIII. (3): The DLR Humanoid 'Rollin' Justin". (4): The DLR-HandII-b, a redesign of the DLR-HandII. (5): The Schunk Hand, a commercialized version of the DLR-HandII. (6): The DLR-Crawler, a walking robot based on the fingers of the DLR-HandII.

pected performance and safety. In this paper we will address the two approaches followed at DLR for reaching the aforementioned new design goals. The first one is the meanwhile mature technology of torque controlled light-weight robots (see Fig.1) . Several products resulted from this research and are currently being commercialized through cooperations with various industrial partners (DLR-KUKA Light-Weight Robot LWRIII, DLR-HIT-Schunk Hand, DLR-Brainlab-KUKA medical robot). The second technology, currently a topic of very active research in the robotics community, is variable compliance actuation. It aims at enhancing the soft robotics features by a paradigm change from impedance controlled systems to variable mechanical stiffness and energy storage, in close interplay with innovative control strategies, as suggested by the human motor system.

Regarding the actively compliant controlled systems, we will concentrate on the newest developments in the design and control leading to the humanoid system Rollin' Justin as well as on the steps required to make the technology widely usable in industrial environments. We are considering these robots as a performance reference, which we are currently trying to outperform with new variable stiffness actuators. We will present the main design ideas and some experimental examples 
providing first validation of the performance and robustness gain of this design approach.

\section{Light-weight, Modular, Torque Controlled Robots}

For almost one decade we focused at DLR on the development of torque controlled, light-weight arms and hands. We refined the technology in successive steps in order to obtain high power actuators, a light-weight though robust design, highly integrated, reliable electronics, and torque sensors with low hysteresis, noise, and drift. Moreover, we developed control algorithms which allow both high performance trajectory tracking and safe and efficient compliant interaction with humans and unknown environments. With the LWRIII and the DLR-Hand IIb a state of maturity and performance of the systems was finally reached, which allowed the commercialization of the two systems in cooperation with industrial partners. The arm is manufactured and distributed by the industrial robot manufacturer KUKA Roboter $\mathrm{GmbH}$, while a simplified version of the hands, designed in cooperation with the Harbin Institute of Technology (China) is distributed by the robot gripper manufacturer Schunk $\mathrm{GmbH}$. Moreover, several spin-off companies emerged from these projects, producing components such as torque sensors and high torque motors. In the last years we started additionally a wide new area of research activities based on this technology by taking advantage of the modular and integrated structure of the components. A fully new line of medical robots was developed, based on both the hand and arm components. The humanoid manipulation system Justin was build up from these components as well, while the modularity of the hands allowed the design of a new crawler robot in only a few months.

In our previous work $[1,2,3,4]$, we presented in detail the design and the control concepts of the LWR-III arm and HandIIb system. In this paper we focus on the evolution of the design and control approaches required for the development of Justin, as well as on the components required for a successful application of the arms in a production assisting environment.

\subsection{Interaction Control of DLR Robotic Systems}

The control of both the arms and hands makes extensive use of the torque sensing available in each joints. The sensors are placed after the gear-box and allow therefore a very precise measurement of the real joint torque, in contrast to simple current based torque estimations. They are, in the given accuracy and sampling rate, a unique feature of the DLR robots, finally turning into reality the old dream of the robotics control community of having robots with torque interface $[5,6]$. The sensors are used to implement both active vibration damping for high performance motion control as well as soft robotics features such as impedance control, collision 
and failure detection, potential field based collision avoidance and posture control. Due to the the relatively high intrinsic compliance of the harmonic drive gears and of the torque sensors, the classical rigid robot assumption is not acceptable for the DLR arms, if high control performance is sought for. Therefore, a major research contribution was to extend many of the known approaches from classical robot control to the flexible joint case by taking advantage of the joint torque measurement. In the flexible joint model, not only the motor position $\theta$, but also the joint torque $\tau$, as well as their derivatives $\dot{\theta}$ and $\dot{\tau}$ are namely states of the system. The measurement of the former and the numerical computation of the latter provides the state estimation required for full state feedback. For the light-weight arm and hands, these methods were presented, e.g., in [1, 2, 3, 4].

The control framework (for both position and impedance control) is constructed from the perspective of passivity theory (Fig. 2) by giving a simple and intuitive physical interpretation in terms of energy shaping to the feedback of the different state vector components.

- A physical interpretation of the joint torque feedback loop is given as the shaping of the motor inertia $B$.

- The feedback of the motor position can be regarded as shaping of the potential energy.

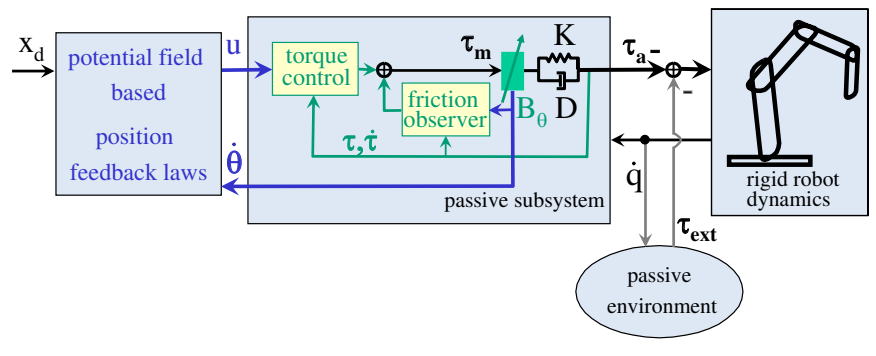

Fig. 2: Representation of passive control structures.

The robustness and performance of the control methods has been extended to product maturity for the commercialization of the light-weight arm in cooperation with KUKA Roboter GmbH and of the Kinemedic/MIRO arms with BrainLab AG. Moreover, during performance tests at the industrial robot manufacturer it turned out that despite of the light-weight, elastic structure, the robot has competitive motion accuracy to an industrial robot of similar payload, according to ISO9283-1998 standard measurements. 


\subsubsection{Disturbance Observers}

Since the control of the DLR robots is fundamentally relying on accurate models of the robot dynamics, friction torques in the gear-box and external interaction torques (from humans or the environment) are a critical source of errors which have to be estimated correctly. Therefore, a new disturbance observer concept was developed [7, 8]. It allows the independent estimation of friction and external collision torques using the same observer structure by exploiting the joint torques signals $\tau$ (see Fig. 3). The friction observer allows high performance motion control as mentioned in the previous section, while the external torque observer is used for safe human-robot interaction, described in Section 2.5. Moreover, although it has an active integrator action, the friction observer can be analyzed within the passivity framework, thus allowing convergence statements for the entire nonlinear system [9].

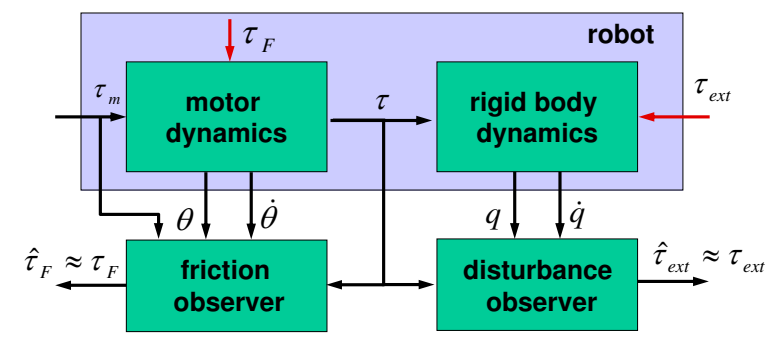

Fig. 3: Disturbance observers for identification of the friction and external interaction torques $\tau_{F}$ and $\tau_{e x t} . \tau_{m}$ and $\tau$ are the motor and the measured torques, respectively.

\subsection{Design and Control of the Humanoid Manipulation System Justin}

Justin was designed as a versatile platform for research on two-handed manipulation and service robotics in everyday human environments. Due to the modular design of the LWRIII as well as of Hand-IIb, it was possible to quickly set up both a lefthanded and right-handed configuration. The robots' common base holds the arms mounted 60 degrees from the vertical in a sideways direction. This allows the elbow to travel fore and aft below the shoulder and up to horizontal height without passing through singularities. To extend the manipulation range, the robot base is held by a four degrees of freedom (DOF) torso. A vertical roll axis, followed by two pitch joints and a third, passive pitch axis which keeps the arm base upright, allows translations in a vertical plane which can rotate about a vertical axis. Through this 
configuration Justin is capable of lifting objects from the floor, reaching over tables and even reaching objects on a shelf of about two meters height (Fig. 4).

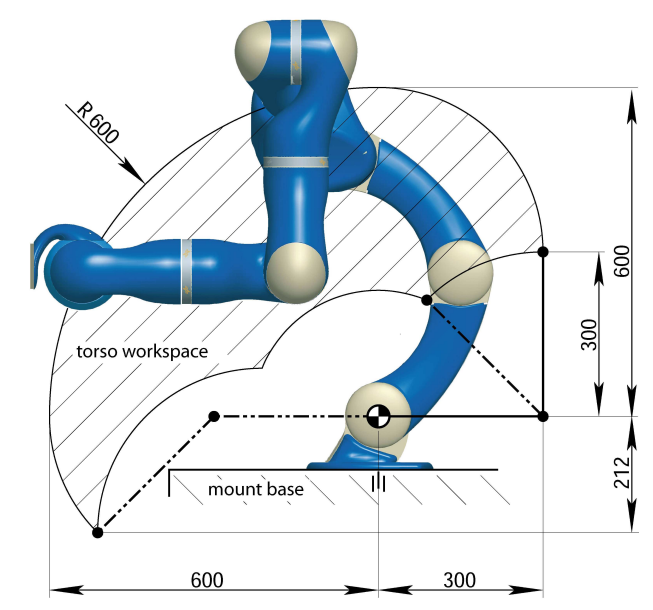

Fig. 4: Workspace design for the humanoid Justin.

To maintain the DLR robot concept, the torso joints consist of the same functional components as the arm joints, allowing full torque control for the setup. In this way, Justin can detect and react to contact forces applied anywhere on its structure.

\subsubsection{Justin's Mobile Plattform}

Justin's new mobile platform enables the system to interact with humans in a larger workspace and thus brings the development towards a universal service robotics platform [10]. The robot base requires a large supporting polygon in order to take advantage of the large workspace, the high forces, and the dynamics of the upper body, while providing the stability of the overall system. On the other hand, compact dimensions are necessary for a reliable and easy navigation through doors or narrow passages. To meet both requirements, our mobile platform has four legs which can be individually extended via parallelogram mechanisms (Fig. 5), even during platform movement. Each leg carries a steerable wheel for omnidirectional (but nonholonomic) movement. This novel kinematics needs new control and planning algorithms [11], since the wheel system has no longer an instantaneous center of rotation while extending or retracting the legs. Furthermore, each leg incorporates a lockable spring damper system. This enables the whole system to drive over small 
obstacles or to cope with the unevenness of the floor, as well as to sustain reaction forces under heavy load. The mobile platform has a weight of $150 \mathrm{~kg}$. Mounted on the mobile platform, Rollin Justin has a shoulder height of up to $1.6 \mathrm{~m}$. The whole system is powered by a Lithium-Polymer battery pack and has an operating time of about $3 \mathrm{~h}$. For enabling the implementation and evaluation of advanced control

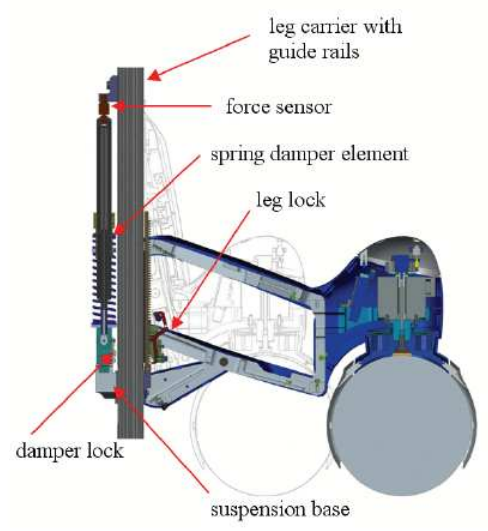

Fig. 5: Variable footprint of Rollin Justins mobile base.

algorithms, the whole upper body is controlled in $1 \mathrm{~ms}$ cycle, while the platform is connected at rate of $16 \mathrm{~ms}$.

\subsubsection{Interaction Control of Justin}

All the interaction control methods developed for the arms and the hands were extended and transferred to Justin in the last three years. The Cartesian impedance controller concept was extended $[12,13]$ to the upper body including hands, arms and the torso (Fig. 6).

Since the mobile platform has only a velocity interface, but no torque interface, a full body compliance control requires to follow an admittance control approach for the base, as sketched in Fig. 7(Right). Therefore, the virtual wrench resulting on base of the torso from the impedance controller of the upper body is transformed using a virtual spring and damper into a velocity command.

In Fig. 7, left, an overview of the entire impedance based control system is shown. A task and trajectory planning stage provides the desired task space motion to the Cartesian impedance controller and a desired posture for the nullspace control. In order to minimize the dynamic reaction forces on the mobile base, a reaction nullspace control approach is integrated into the system [14]. For achieving safety for humans in the workspace of the robot, the system contains two complementary approaches. 


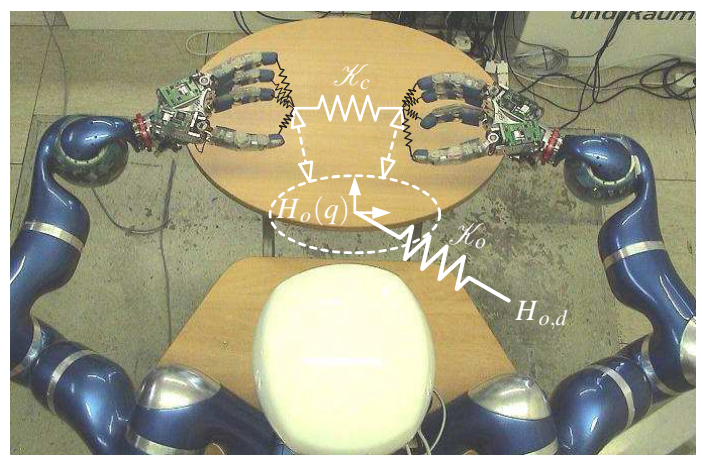

Fig. 6: Two-hand impedance behavior by combining object level impedances of the hands and the arms.
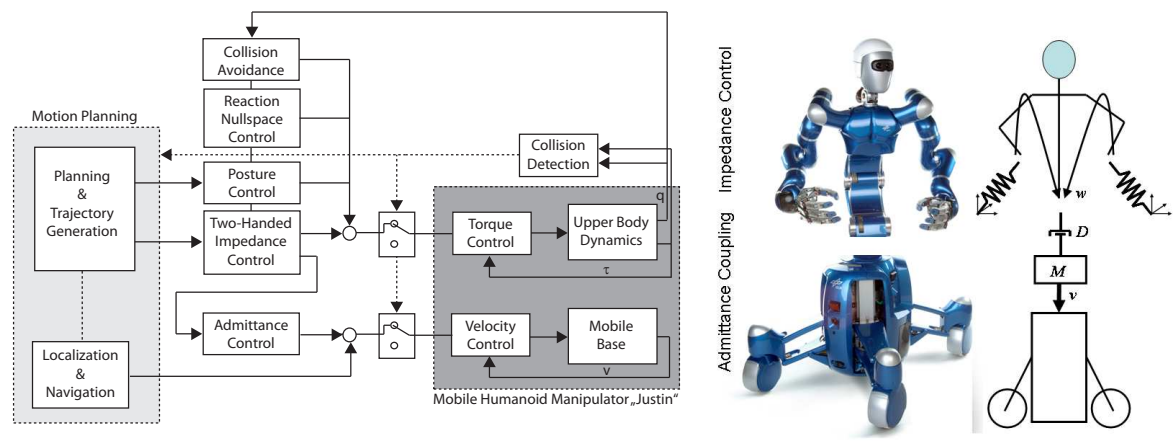

Fig. 7: Left: Torque based Control Structure for Justin. Right: The upper body is impedance controlled in a 1ms cycle. The base is admittance controlled and its desired velocity is related to the virtual force produced by the controller in the base of the torso by a virtual mass-damper dynamics.

Firstly, a potential function based collision avoidance is used [15]. Secondly, a disturbance observer based collision detection routine allows to implement different collision reaction strategies (Sec. 2.5, [8]).

\subsection{Technology transfer: Compliant Industrial Assistant}

As a result of the technology transfer to KUKA Roboter GmbH, the KUKA lightweight robots are currently used in numerous academic and industrial research labs. The new automation concepts based on this robot allow higher flexibility due to fast work-cell setup and modification, intuitive hands-on programming, and shared workspace for direct interaction and cooperation of humans and robots. The first industrial application was realized by the Daimler factory automation department in 

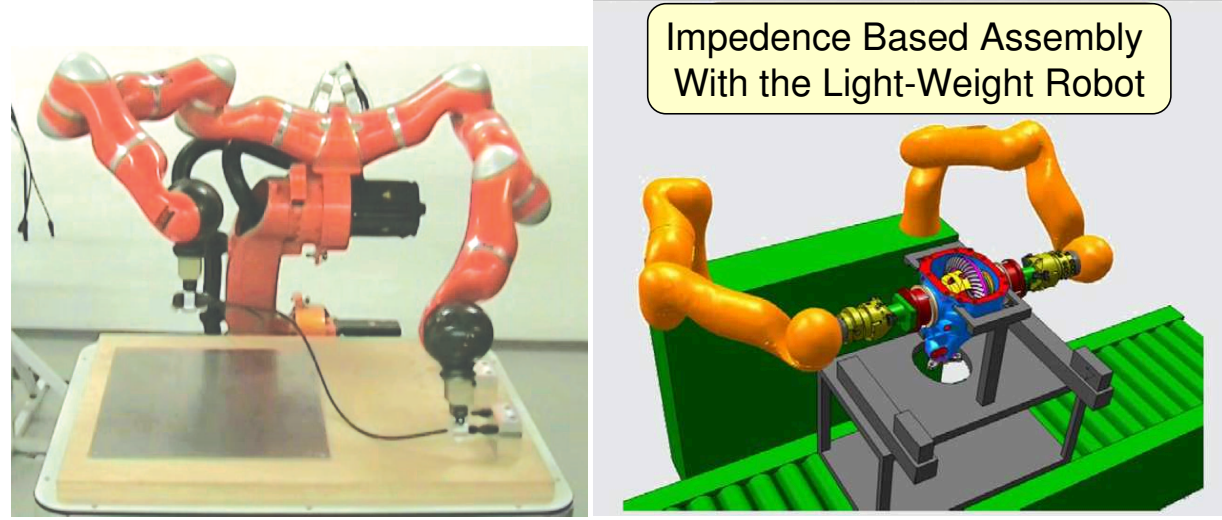

Fig. 8: Left: Demonstration of bimanual flexible object handling by KUKA Roboter GmbH. Right: Impedance based two arm assembly in Mercedes car manufacturing. Courtesy Daimler AG.

Untertürkheim. The system is now used for automatic gear-box assembly in daily production (Fig.8).

In order to establish the new technology in industrial environments, two further key aspects need to be addressed:

- The programmer has to be supported with appropriate toolboxes which help to use and parameterize the various control features of the robot, such as compliance, center of compliance, damping, assembly path, collision detection and reaction strategy, or controller switch for a given application.

- The safety of humans during the permanent interaction with the robots always has to be ensured. The new field of robotic safety in human-robot interaction requires research in biomechanics for understanding injury mechanisms as well as methods for preventing or reducing them.

These two topics are addressed in the next sections.

\subsection{Planning Toolbox for Impedance Based Automatic Assembly}

Assembly is one of today's the most demanding tasks for industrial robots. Parts have to be brought into contact and aligned properly by the robot despite inevitable uncertainties due to part tolerances, imprecise part feeding and limited robot positioning accuracy. Lack of robustness, extensive setup costs for high-precision part feeding, specialized grippers with so-called Remote Center Compliance, and the need for experienced robot programmers are the main reasons, why most assembly tasks are still carried out by humans. 

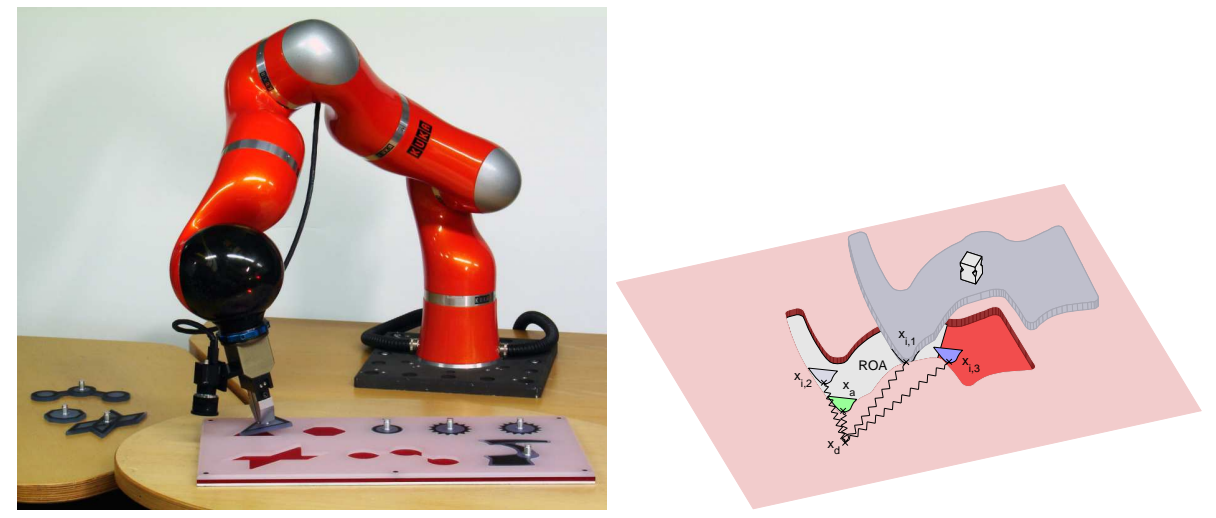

Fig. 9: Left: Experimental setup consisting of a DLR light-weight robot with an industrial gripper, an attached Firewire camera and the pieces and plate on the table. Right: A typical region of attraction (ROA) for a sample part. The inserted corner will be guided automatically to position $x_{a}$ if the alignment process starts anywhere within the ROA (e.g. from $x_{i, 1}$ or $x_{i, 2}$ ). If it starts outside (e.g. from $x_{i, 3}$ ), a successful alignment cannot be guaranteed.

In contrast to current industrial robots, the compliant control features of the DLR light-weight robot allow flexible and robust assembly without additional equipment. The programmer can select high-performance position control for free motion and compliant Cartesian impedance control for highly dynamical interaction with the environment. If desired, the switching between controllers can be triggered by contact detection within $1 \mathrm{~ms}$.

Along with stable contact control, proper alignment of the parts despite inevitable uncertainties, is the most challenging part of an assembly task. Usually, this requires tedious and expensive manual optimization of the trajectories for every type of object. In order to simplify this procedure, an algorithm has been developed, which allows automatic planning of robust assembly applications. The algorithm takes the part geometries and information about the expected uncertainties as an input and generates a parameterized robot program for the robust assembly of the parts [16].

The main idea of the insertion planning is visualized in Fig. 9 (Right). Consider the compliance controlled robot having inserted a corner ${ }^{1}$ of the part into the hole at the initial configuration $x_{i}$. The desired position of the controller is now set to $x_{d}$, and the stiffness value to $K$. For a certain set of starting configurations (called the region of attraction - $\mathrm{ROA}$ ), the inserted part will converge to the desired alignment position $x_{a}$. In the given example, $x_{i, 1}$ and $x_{i, 2}$ belong to the ROA, $x_{i, 3}$ does not. The alignment can be seen as the settling of a nonlinear dynamic system with several equilibria, whereof one is the desired configuration. It is possible to determine the ROA for any desired equilibrium $x_{d}$ and for any stiffness matrix $K$. Its size can be

${ }^{1}$ Corner in this context means the relevant part of the contour which is involved in a one-point contact. 
used as a direct measure for the robustness of the assembly trajectory. The optimal robustness is achieved for those insertion parameters that maximize the ROA.

Obviously, the ROA depends heavily on the inserted corner, the selected desired and initial positions $x_{d}$ and $x_{i}$, the parameters of the impedance control (in particular $K$ ), and the shape of the hole. Whereas the latter is given, the remaining parameters can be freely selected and are used for offline optimization. Combined with a user interface for providing the geometries from a CAD system or from sensor data, this toolbox for industrial robot programmers generates robust assembly programs automatically. The output of the toolbox, desired trajectories and control parameters, can then be used in the execution phase without any model knowledge of the parts.

The robustness and performance of the generated assembly strategies were evaluated in extensive experiments with parts having a clearance of less than $0.1 \mathrm{~mm}$ [17]. The parts are freely placed on a table, located with appropriate image processing, and approached via visual servoing. In order to assess the performance, a comparison with humans in terms of execution time was done. Altogether, 41 persons were tested, whereof 35 were children of age 5-7 and the remaining were adults.

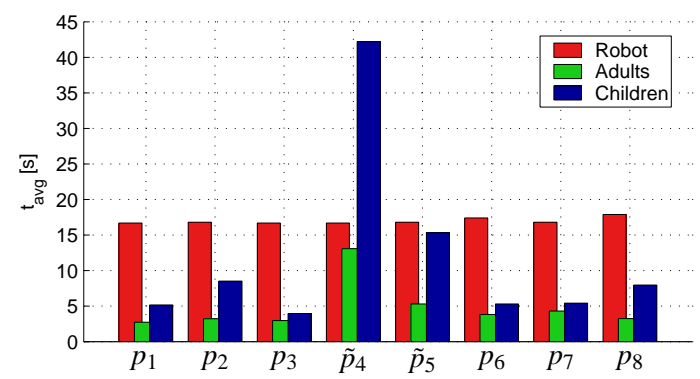

Fig. 10: Average times needed for the different parts. Whereas the robot shows similar performance for all the parts, humans have difficulties especially with the differentiation and insertion of the star shapes as those are difficult to distinguish for humans. $\tilde{p}_{4}$ represents the star that is inserted first (can be $p_{4}$ or $p_{5}$ ), $\tilde{p}_{5}$ the other one.

Adults needed roughly $30 \%$ of the robot's total time for the eight given parts, while children needed about $70 \%$. The variation of the robot performance was low, since the only nondeterministic part of the strategy was the pieces searching (see Fig. 10). Humans, instead, varied their strategy, trying first to solve the problem as fast as possible (accepting failure), and then refined the strategy in subsequent attempts if necessary. Some children needed considerably longer than the robot and were able to fulfil the task only with additional hints.

While free motion and part picking of the humans was considerably faster, in average the robot performed better and more constant in the insertion phase. The experiment shows that the combination of global vision and local force information can be considered as a key to robust and flexible industrial assembly tasks. 


\subsection{Safety in Human-Robot Interaction}

An essential requirement for a robot designed for direct interaction with human users, as e.g. for production assistants, is that it must in no case pose a threat to the human. Until recently, quite few attempts were made to investigate real world threats via collision tests and use the outcome for considerably improving safety during physical human-robot interaction. In this section, we give a brief overview of our systematic evaluation of safety in human-robot interaction with emphasis on aspects related to the LWRIII.

\subsubsection{Standardized Crash Tests Experiments for Blunt Impacts}

In $[18,19,20,21,22]$ we analyzed and quantified impact characteristics of blunt robot-human collisions and significantly augmented existing knowledge in this field. The results were obtained and verified with standardized equipment from automobile crash testing, leading to an extensive crash-test report for robots of different size and weight. They range from the LWRIII to heavy duty industrial robots [23, 24]. For the LWRIII all impact tests generated very low injury values by means of standardized severity indices evaluated for the head, neck, and chest. The Head Injury Criterion ${ }^{2}$ reached a maximum numerical value of 25 at $2 \mathrm{~m} / \mathrm{s}$, which is equivalent to $\approx 0 \%$ probability of injury by means of HIC. For both neck and chest similar conclusions could be drawn, since all injury measures were far below any safety critical value [18]. These results were confirmed by impact tests with a human [22]. Even for the case of clamping close to a singularity, which turned out to be the worst-case for the LWRIII, the robot was not able to produce large enough forces to break the frontal bone or endanger the chest of a human, though producing a high quasi-static force of $\approx 1.6 \mathrm{kN}$.

Apart from such worst-case analysis, we developed effective collision detection and reaction schemes for the LWRIII using the joint torque sensors [25, 26], (see Sec. 2.1.1), which proved to be very effective to reduce the injury potential. Even for the afore-mentioned difficult case ${ }^{3}$ we could experimentally verify a reduction of the contact force down to $\approx 500 \mathrm{~N}$ for the almost outstretched case. This significantly relaxes the theoretical results of [22].

An important outcome of the extensive experimental campaign is that generally blunt dynamic impacts in free space are, regardless the mass, not dangerous up to an impact velocity of $2 \mathrm{~m} / \mathrm{s}$ with respect to the investigated severity indices. On the other hand, impacts with (partial) clamping can be lethal, significantly depending on the robot mass. This led us to recommendations for standardized crash-testing procedures in robotics, c.f. Fig. 11. The proposed impact procedures can hopefully

\footnotetext{
2 The Head Injury Criterion (HIC) is the injury severity criterion best known in robotics. Intuitively speaking, a value of 650 corresponds to a $5 \%$ probability of staying one day in hospital, while a value of over 1000 can be lethal.

${ }^{3}$ Due to the almost singular configuration, the joint torque sensors are quite insensitive to the clamping force.
} 

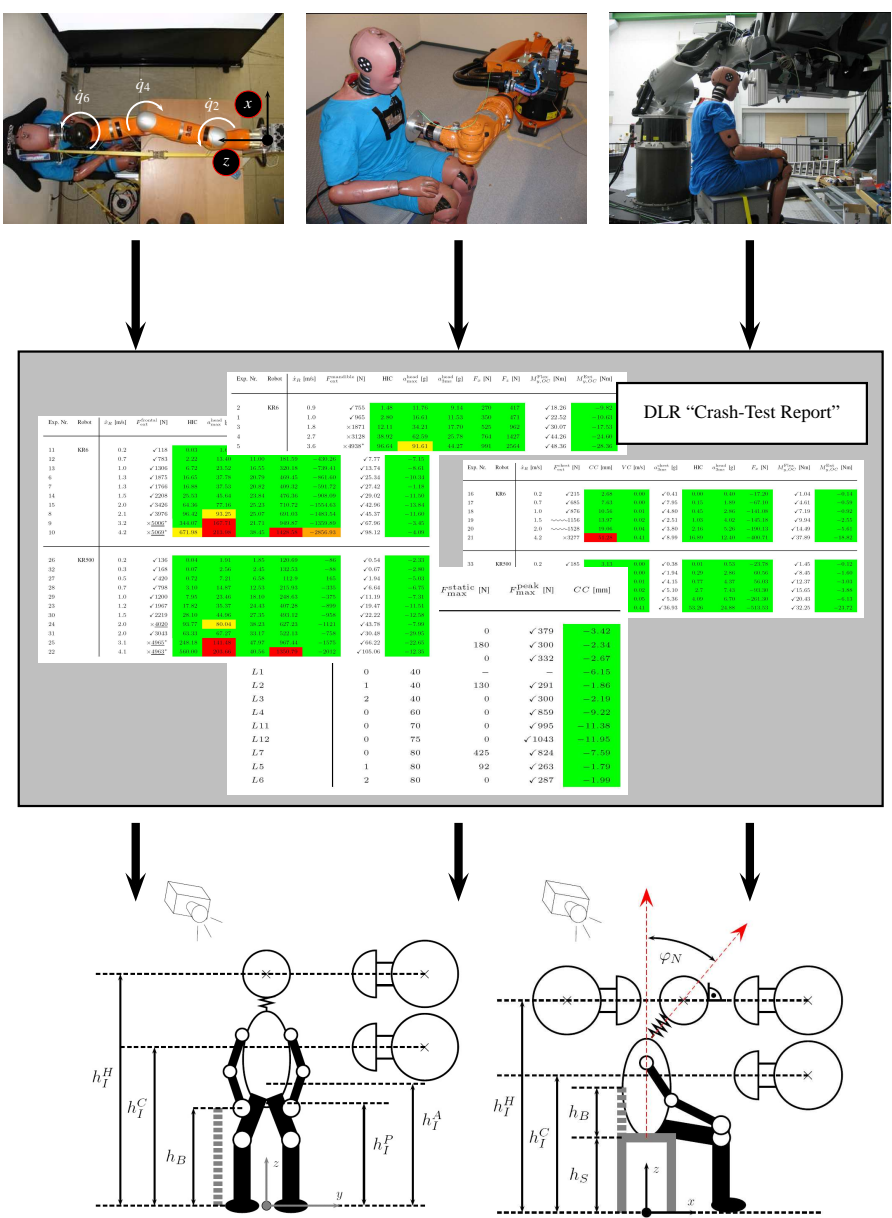

Fig. 11: From impact testing with standardized equipment and evaluation of biomechanical injury criteria to a proposal of standardized impact testing in robotics.

provide substantial contributions for future safety standards in physical human-robot interaction.

Apart from blunt impacts, it is of immanent importance to treat soft-tissue injury due to sharp contact. 

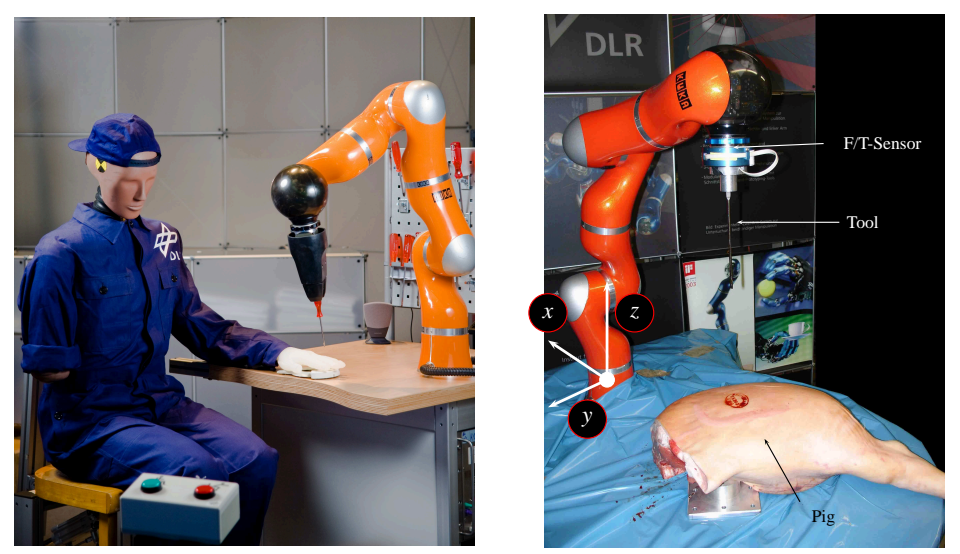

Fig. 12: The co-worker scenario is an example of a robot, which is potentially equipped with dangerous tools, interacting with humans (left). Testing setup for the pig experimental series (right).

\subsubsection{Soft-tissue Injury Caused by Sharp Tools}

A major potential injury source in pHRI are the various tools a robot can be equipped with, see Fig. 12 (left). Their evaluation is still a field with numerous open issues and definitely worth and fruitful to work on. As a first step, we were able to identify the most important injuries and their causes, based on investigations made in the field of forensic medicine and biomechanics. In [27] we presented various experimental results with biological tissue, which validate the analysis. Furthermore, an evaluation of possible countermeasures by means of collision detection and reaction is carried out, c.f. Fig. 12 (right).

It was possible to detect and react to stabbing impacts at $0.64 \mathrm{~m} / \mathrm{s}$ fast enough to limit the penetration (e.g., of a knife) to subcritical values of several mm's or even prevent penetration entirely, depending on the tool. In case of cutting a full prevention of penetration at a velocity of $0.8 \mathrm{~m} / \mathrm{s}$ was achieved. Furthermore, we found empirically relevant safety limits for injury prevention for the case of sharp contact, as e.g. the skin deformation before penetration.

\section{Increased Performance and Robustness trough Variable Impedance Actuation}

Based on the experience gained with the very successful approach of torque controlled robots, we identified also its limitations and addessed new directions of research for further increasing the robustness, performance and safety of robots. A comparison between actual service robots and their human archetype still shows large discrepancy in several aspects. Firstly, relatively small impacts can cause se- 
vere damages to a robot. The DLR arms and hands are close to their gear-box torque limits when catching a ball of $80 \mathrm{~g}$ having a speed of $28 \mathrm{~km} / \mathrm{h}$ while for instance a handball goal keeper easily withstands a hit at $120 \mathrm{~km} / \mathrm{h}$ of a $425 \mathrm{~g}$ ball. In the second case, the impact energy is 100 times larger than in the first case. The "as stiff as possible" mechanical design paradigm and the torque control reach their limits here, because the impact lasts typically only few milliseconds for such a robot. This is too short for the actuator to react and accelerate the motor and gear-box for reducing the impact. This shows that the robustness of robots against impacts can not be addressed by further improvements of torque controlled robots but needs a change of paradigm. The motor has to be partially decoupled from the link side and the induced energy must be stored within the robot joint instead of being dissipated. This directly leads to the necessity of passive elastic elements.

Another important observation is that the velocity and dynamic force capabilities of current robots are by far not good enough to perform dynamic tasks, such as throwing and running, as good as human beings. This can also be improved by the use of mechanical energy storage within the system as exemplified in Sec. 3.3.

Since the specifications for several tasks vary widely regarding position accuracy, speed, and required stiffness, the joint stiffness needs to be variable. This requires an additional motor per joint. To keep the drawbacks of having a second actuator at each joint as low as possible, the joint unit has to be optimized regarding its energy efficiency e.g. at high stiffness presets. The concept of variable impedance actuation $^{4}$ (VIA) seems to be a promising solution in this context and its design and control was addressed in numerous publications [28, 29, 30, 31, 32].

Our goal is, based on our experience with torque controlled light-weight robots, to built up a fully integrated VIA hand-arm system for close, safe, high performance interaction with humans while fulfilling the above requirements as close as possible (Fig. 13).

\subsection{Design of Variable Stiffness Systems}

Currently, a hand-arm system with variable compliance is designed at DLR incorporating in a first, concept validation version, several variable compliance joint designs for fingers and arms, see Fig. 13. For the hand, an antagonistic approach is taken, which allows to place the actuators and the variable stiffness mechanics in the forearm and to transmit the motion via tendons through the wrist to the fingers. The fingers and the hand structure are designed to match as close as possible the human hand kinematics and functionality, while finding innovative technological solutions for their implementation [33] (Fig. 13). The wrist is also actuated antagonistically, however in a supporting setup. In such a setup both motors can add their torques to gain the maximum possible torque output or can co-contract to change stiffness for medium load. For the elbow and the shoulder, the focus is on energy efficient and

${ }^{4}$ If the joint has only variable stiffness, but no variable damping, the term variable stiffness actuation (VSA) is often used. 

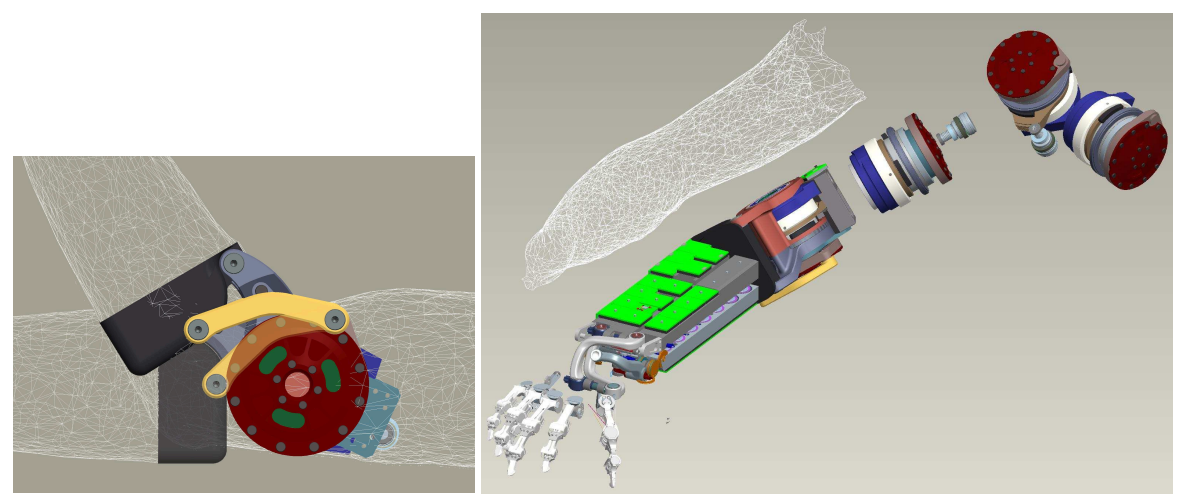

Fig. 13: Current stage of the DLR VIA hand-arm system. Left: Elbow joint. Right: Explosion drawing of the hand-arm system.

weight minimizing design, such that the mass of the VIA joints do not considerably exceed the weight of an LBWRIII joint. The actuators of these joints are based on

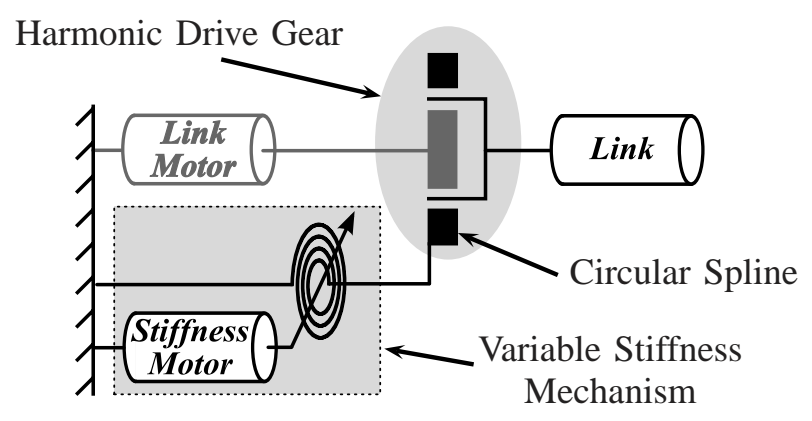

Fig. 14: Actuator and compliance arrangement for the shoulder and elbow joints.

an approach in which a small motor is primarily used to adapt the stiffness of the joint and a large motor is mainly used to position the link (Fig.14). The currently followed design is a combination of quasi-antagonistic and the variable stiffness joint designs (Fig.15) presented previously in [34, 13].

\subsection{Control Challenges with VIA actuators}

The classical control problem formulation for VSA robots is that of adjusting stiffness and position of one actuator and of the entire robotic system (arm, hand) in a decoupled manner, by controlling the position or the torque of the two motors of 

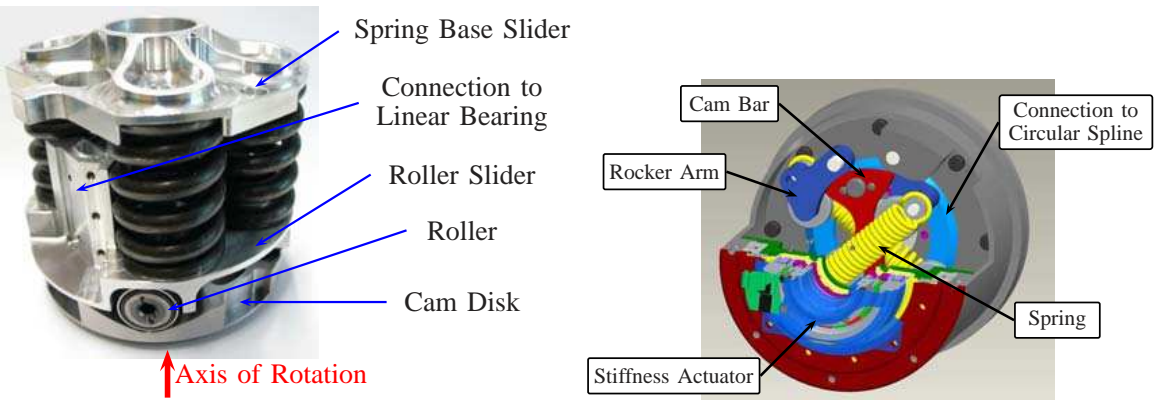

Fig. 15: Design versions of the shoulder and elbow joints. Left: Variable Stiffness Actuator. Right: Quasi-Antagonistic Joint.

the actuator [31, 32, 29]. Moreover, in case of VSA structures with many DOF and cable actuation, the decoupling tendon control is treated [35, 36].

In [37] we proposed a new solution for the design of impedance control for coupled tendon systems with exponential stiffness (Fig. 16). The proposed controller provides statica decoupling of position and stiffness as well as the exact desired link side stiffness in combination with the intrinsic mechanical compliance, while remaining within the passivity framework of the DLR robots. A second challenging

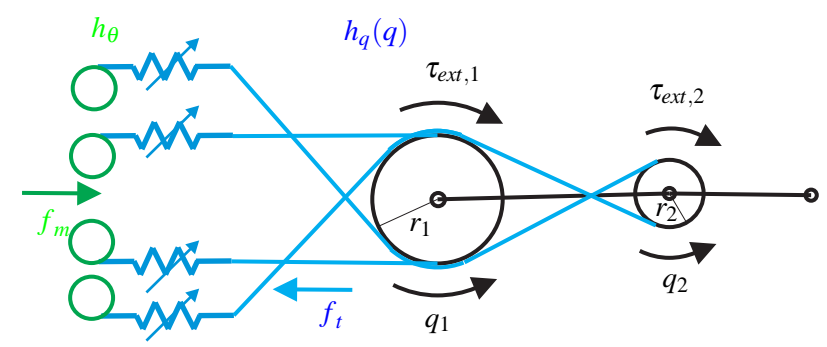

Fig. 16: Example of a tendon network with two joints and four tendons connected by nonlinear springs. $h_{\theta}$ and $h_{q}$ are the motor and link side tendon displacements, respectively, $f_{m}$ and $f_{t}$ are the motor and link side tendon forces.

control task is related to the fact that almost all VIA joints designed so far have very low intrinsic damping. While this feature is very useful for movements involving energy storage (e.g. for running or throwing), the damping of the arm for fast, fine positioning tasks has to be realized by control. This can be a difficult task, regarding the strong variation of both inertia and stiffness. Fortunately, the passivity based approaches developed for the torque controlled robots can be adapted for the VIA case. However, it soon became clear from the simulation for the whole arm that a separate control of each joint, by just considering diagonal components of stiffness 
and inertia matrices as inputs, is not feasible, due to very low stiffness and strong coupling between the compliant joints. New methods for treating the joint coupling were developed starting from $[38,1]$. The basic idea for the controller design is the following:

- Consider full coupled inertia and stiffness matrices for the relevant joints.

- Transform the system consisting of link inertia and stiffness to modal coordinates such that the two matrices become diagonal.

- Use torque feedback in order to bring the motor inertia matrix to a structure in correspondence to the double diagonalized matrices, i.e. make it diagonal in the same coordinates.

- Design a decoupled controller in the modal coordinates, independently for each mode. Gains are calculated based on current modal parameters.

With this methods, the control proved to work well, as exemplified in the plots from Fig. 17, for one of the three joints. An experimental validation of the controller for high an low stiffness preset on a 1 DOF testbed is shown in Fig. 18.
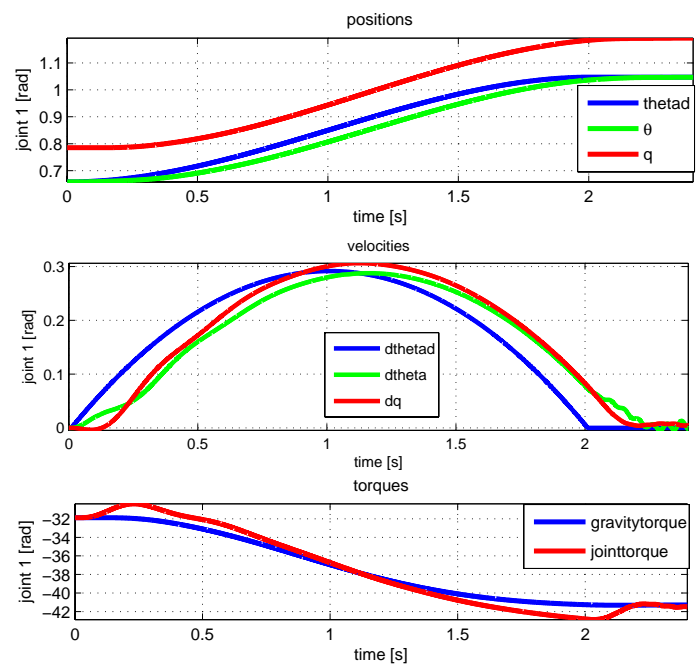

Fig. 17: Motor and link position with state feedback controller.

\subsection{Validation of Performance and Robustness}

Along with the activities regarding the control of the joint, first analysis and experiments for validating the increase in performance were done. 

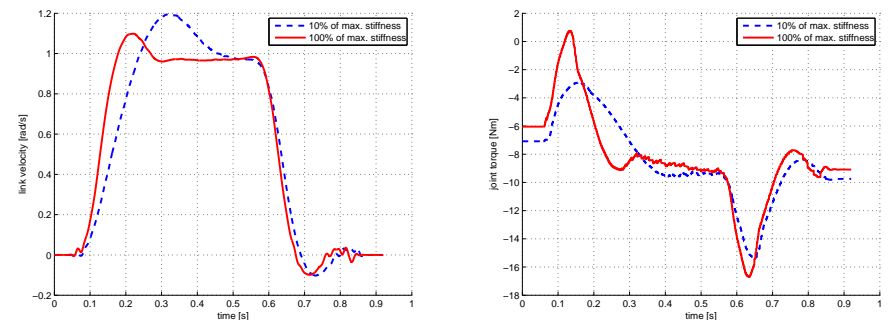

Fig. 18: Motion on a trajectory with rectangular velocity profile for small and maximal stiffness. A critically damped velocity step response can be achieved independent from the stiffness and inertia value (upper). The effect of vibration damping is clearly observed in the torque signal (lower).

\subsubsection{Throwing}

The application of throwing a ball is a good example to show the performance enhancement gained by the VS-Joint in terms of maximal velocity. For throwing a ball as far as possible, it has to be accelerated to the maximum achievable velocity and released at an angle of $45^{\circ}$. The link velocity of a stiff link corresponds to the velocity of the driving motor. In a flexible joint the potential energy stored in the system can be used to accelerate the link relatively to the driving motor. Additional energy can be inserted by the stiffness adjuster of the variable stiffness joint to gain an even faster motion.

Fig. 19 shows simulation results and experimental validation regarding the velocity gain between motor and link for the quasi-antagonistic link. The motor trajectories for optimal performance were generated by an optimal control approach [39]. The link velocity is maximized under constraints on motor velocity and torque, elastic joint deflection range, controller dynamics.

With the measured maximum link velocity of $572^{\circ} \mathrm{s}^{-1}$, the throwing distance for the same experiment with the Variable Stiffness Joint was approximately $6 \mathrm{~m}$, corresponding well to the calculated distance of $6.18 \mathrm{~m}$. The theoretical throwing distance with an inelastic link of the same setup with the same maximum motor velocity of $216^{\circ} \mathrm{s}^{-1}$ is $0.88 \mathrm{~m}$, also was confirmed experimentally. A speed gain of $265 \%$ for the link velocity between rigid and compliant joint was achieved in the test. Similar results in performance increase have been obtained for kicking a soccer ball, which additionally causes an external impact on the link side, as discussed next.

\subsubsection{Experimental Validation of Joint Overload Protection at Impacts}

In [40], two series of experiments were conducted to investigate the benefits of passive variable stiffness during impacts. The testing setup for both series was a 

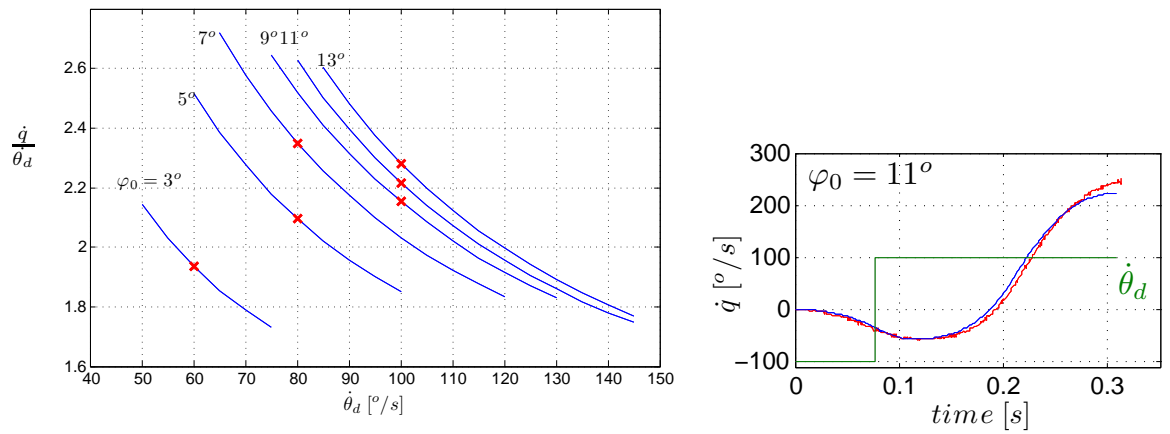

Fig. 19: Left: blue - simulated gain in velocity between motor and link, depending on the maximal desired motor velocity and the stiffness preset. Red - experimentally validated points. Right: Desired motor velocity (grey) and reached link velocity for one experiment (red-simulated, bluemeasured)

single DOF joint (with link inertia $\approx 0.57 \mathrm{~kg} \mathrm{~m}^{2}$ ) being hit at a lever length of $\approx$ $0.76 \mathrm{~m}$ by a soccer ball $(0.45 \mathrm{~kg})$.

In the first series the unloaded joint is kept still and passively hit by the ball with different impact speeds. The joint torques were recorded for three different setups. Two stiffness setups are realized via the passively compliant VS-Joint. The most compliant as well as the stiffest configuration were chosen. In a third setup a mechanical shortcut is inserted into the test-bed instead of the VS-Joint mechanism, such that a much stiffer joint in the range of the LWRIII elasticity is obtained. Both,

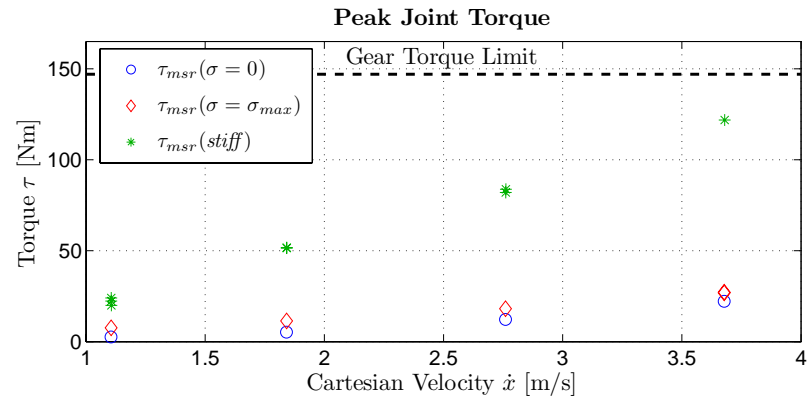

Fig. 20: Peak joint torque during impacts with with a soccer ball. Three different stiffness setups are examined: VS-Joint at low stiffness preset, VS-Joint at high stiffness preset, and an extremely stiff joint without deliberate elasticity (upper). Higher impact velocities result in larger peak torque and passive joint deflection.

increasing impact speed and increasing joint stiffness result in higher peak joint torques as visualized in Fig. 20. The peak torque limit of the joint gear is almost 
reached with the stiff joint at an impact velocity of $\approx 3.7 \mathrm{~m} / \mathrm{s}$, whereas the compliant VS-Joint is still far in the safe torque region.

In the second test series the resting soccer ball was hit by the joint lever at maximum joint velocity. In case of the stiff joint the velocity is limited by the motor. With the VS-Joint, the joint velocity was increased by the energy storage in the joint with a similar trajectory to the one used in Sec. 3.3.1. The results given in Table 1 show a significant increase in joint velocity and kicking range with the VS-Joint which results in a faster impact on the ball. The tests show, however, that the peak joint torque is much smaller in the flexible joint even though the impact was faster. So the passive flexibility in the VS-Joint does not only help to increase the joint performance, but also reduces the potentially harmful peak joint torques during fast rigid impacts.

\begin{tabular}{lccr}
\hline \multicolumn{3}{l}{ Joint Type Joint Velocity } & Peak Joint Torque Kicking Range \\
\hline Stiff Joint & $229 \mathrm{deg} / \mathrm{s}$ & $85 \mathrm{Nm}$ & $1.6 \mathrm{~m}$ \\
\hline VS-Joint & $475 \mathrm{deg} / \mathrm{s}$ & $10 \mathrm{Nm}$ & $4.05 \mathrm{~m}$ \\
\hline
\end{tabular}

Table 1: Results for the different kicking impacts for the VS-Joint and for the rigid joint.

\section{Summary}

This paper presented a bird's-eye-view of the paradigm evolution from high performance torque controlled robots to systems with intrinsic variable stiffness. We overviewed the major design and control principles of the torque controlled robot systems developed at the German Aerospace Center (DLR) as an antetype. Torque controlled robots currently represent a technology that is mature for the market. They are used not only as a tools for academic research but also in industrial environments, within new, more flexible automation concepts based on direct cooperation of robots and humans. We believe, however, that impressive research progress can be expected in the area of VSA actuated robots within the next decade. The motivation for variable impedance devices, derived from different performance, robustness, and safety requirements, are highlighted. Possible hardware solutions are described, which are currently investigated for a newly developed hand-arm system at DLR. Finally, first experimental results validating these concepts were presented.

\section{References}

1. A. Albu-Schäffer, Ch. Ott, and G. Hirzinger, "A unified passivity based control framework for position, torque and impedance control of flexible joint robots," The Int. J. of Robotics Research, vol. 26, no. 1, pp. 23-39, 2007.

2. Ch. Ott, A. Albu-Schäffer, A. Kugi, and G. Hirzinger, "On the passivity based impedance control of flexible joint robots," IEEE Transactions on Robotics and Automation, vol. 24, no. 2 , pp. $416-429,2008$. 
3. A. Albu-Schäffer, S. Haddadin, Ch. Ott, A. Stemmer, T. Wimböck, , and G. Hirzinger, "The DLR lightweight robot - design and control concepts for robots in human environments," Industrial Robot: An International Journal, vol. 134, no. 5, 2007.

4. T. Wimböck, Ch. Ott, and G. Hirzinger, "Passivity-based object-level impedance control for a multifingered hand," in IEEE/RSJ International Conference on Intelligent Robots and Systems, 2006, pp. 4621-4627.

5. L. E. Pfeffer, O. Khatib, and J. Hanke, "Joint-torque sensory feedback in the control of a puma manipulator," IEEE Trans. Robot. Automation, vol. 5, pp. 418-425, 1989.

6. O. Khatib, "A unified approach for motion and force control of robot manipulators: The operational space formulation," $R A$, vol. RA-3, pp. 43-53, 1987.

7. A. De Luca, A. Albu-Schäffer, S. Haddadin, and G. Hirzinger, "Collision Detection and Safe Reaction with the DLR-III Lightweight Manipulator Arm," IEEE/RSJ Int. Conf. on Intelligent Robots and Systems (IROS2006), Beijing, China, pp. 1623-1630, 2006.

8. S. Haddadin, A. Albu-Schäffer, A. De Luca, and G. Hirzinger, "Collision detection \& reaction: A contribution to safe physical human-robot interaction," IEEE/RSJ Int. Conf. on Intelligent Robots and Systems, pp. 3356-3363, 2008.

9. L. L. Tien, A. Albu-Schffer, A. D. Luca, , and G. Hirzinger, "Friction observer and compensation for control of robots with joint torque measurement," IEEE/RSJ Int. Conf. on Intelligent Robots and Systems, pp. 3789-3795, 2008.

10. M. Fuchs, Ch. Borst, P. Robuffo-Giordano, A. Baumann, E. Kraemer, J. Langwald, R. Gruber, N. Seitz, G. Plank, K. Kunze, R. Burger, F. Schmidt, T. Wimbck, and G. Hirzinger, "Rollin Justin - design considerations and realization of a mobile platform for a humanoid upper body," IEEE Int. Conf. on Robotics and Automation, vol. 22, pp. 4131-4137, 2009.

11. P. Robuffo-Giordano, A. Albu-Schäffer, M. Fuchs, and G. Hirzinger, "On the kinematic modeling and control of a mobile platform equipped with steering wheels and movable legs," IEEE Int. Conf. on Robotics and Automation, 2009.

12. T. Wimböck, Ch. Ott, and G. Hirzinger, "Impedance behaviors for two-handed manipulation: Design and experiments," in IEEE International Conference on Robotics and Automation, 2007, pp. 4182-4189.

13. A. Albu-Schäffer, O. Eiberger, M. Grebenstein, S. Haddadin, Ch. Ott, and G. H. T. Wimbock, S. Wolf, "Soft robotics: From torque feedback controlled lightweight robots to intrinsically compliant systems," IEEE Robotics \& Automation Magazine, pp. 20 - 30, 2008.

14. T. Wimböck, D. Nenchev, A. Albu-Schäffer, and G. Hirzinger, "Experimental study on dynamic reactionless motions with DLR's humanoid robot Justin," IEEE/RSJ Int. Conf. on Intelligent Robots and Systems, 2009.

15. A. de Santis, A. Albu-Schäffer, C. Ott, B. Siciliano, and G. Hirzinger, "The skeleton algorithm for self-collision avoidance of a humanoid manipulator," IEEE/ASME International Conference on Advanced Intelligent Mechatronics, 2007.

16. A. Stemmer, A. Albu-Schäffer, and G. Hirzinger, "An analytical method for the planning of robust assembly tasks of complex shaped planar parts," Proc. 2007 IEEE Int. Conf. of Robotics and Automation, pp. 317-323, 2007.

17. P. Robuffo-Giordano, A. Stemmer, K. Arbter, and A. Albu-Schäffer, "Robotic assembly of complex planar parts: An experimental evaluation," IROS, pp. 3775 - 3782, 2008.

18. S. Haddadin, A. Albu-Schäffer, and G. Hirzinger, "Safety Evaluation of Physical HumanRobot Interaction via Crash-Testing," Robotics: Science and Systems Conference (RSS2007), Atlanta, USA, pp. 217-224, 2007.

19. _ - "The Role of the Robot Mass and Velocity in Physical Human-Robot Interaction - Part I: Unconstrained Blunt Impacts," in IEEE International Conference on Robotics and Automation (ICRA2008), Pasadena, USA, 2008, pp. 1331-1338.

20. — , "The Role of the Robot Mass and Velocity in Physical Human-Robot Interaction - Part II: Constrained Blunt Impacts," IEEE Int. Conf. on Robotics and Automation (ICRA2008), Pasadena, USA, pp. 1339-1345, 2008.

21. —_ "Safe Physical Human-Robot Interaction: Measurements, Analysis \& New Insights," in International Symposium on Robotics Research (ISRR2007), Hiroshima, Japan, 2007, pp. 439-450. 
22. — - "Requirements for safe robots: Measurements, analysis, \& new insights," accepted at: International Journal of Robotics Research, 2009.

23. S. Haddadin, A. Albu-Schäffer, M. Frommberger, J. Rossmann, and G. Hirzinger, "The "DLR Crash Report": Towards a Standard Crash-Testing Protocol for Robot Safety - Part I: Results," in IEEE Int. Conf. on Robotics and Automation (ICRA2008), Kobe, Japan, 2009, pp. 272-279.

24. — "The "DLR Crash Report": Towards a Standard Crash-Testing Protocol for Robot Safety - Part II: Discussions," IEEE Int. Conf. on Robotics and Automation (ICRA2008), Kobe, Japan, pp. 280-287, 2009.

25. A. De Luca, A. Albu-Schäffer, S. Haddadin, and G. Hirzinger, "Collision Detection and Safe Reaction with the DLR-III Lightweight Manipulator Arm," in IEEE/RSJ Int. Conf. on Intelligent Robots and Systems (IROS2006), Beijing, China, 2006, pp. 1623-1630.

26. S. Haddadin, A. Albu-Schäffer, A. D. Luca, and G. Hirzinger, "Collision detection \& reaction: A contribution to safe physical human-robot interaction," in IEEE/RSJ Int. Conf. on Intelligent Robots and Systems (IROS2008), Nice, France, 2008, pp. 3356-3363.

27. S. Haddadin, A. Albu-Schäffer, A. De Luca, and G. Hirzinger, "Evaluation of Collision Detection and Reaction for a Human-Friendly Robot on Biological Tissue," in IARP International Workshop on Technical challenges and for dependable robots in Human environments (IARP2008), Pasadena, USA, 2008. [Online]. Available: www.robotic.de/Sami.Haddadin

28. T. Morita, H. Iwata, and S. Sugano, "Development of human symbiotic robot: Wendy." IEEE Int. Conf. of Robotics and Automation, pp. 3183-3188, 1999.

29. A. Bicchi and G. Tonietti, "Fast and Soft Arm Tactics: Dealing with the Safety-Performance Trade-Off in Robot Arms Design and Control," IEEE Robotics and Automation Mag., vol. 11, pp. 22-33, 2004.

30. S. A. Migliore, E. A. Brown, and S. P. DeWeerth, "Biologically Inspired Joint Stiffness Control," in IEEE Int. Conf. on Robotics and Automation (ICRA2005), Barcelona, Spain, 2005.

31. G. Palli, C. Melchiorri, T. Wimboeck, M. Grebenstein, and G. Hirzinger, "Feedback linearization and simultaneous stiffness-position control of robots with antagonistic actuated joints," in IEEE Int. Conf. on Robotics and Automation (ICRA2007), Rome, Italy, 2007, pp. 2928-2933.

32. B. Vanderborght, B. Verrelst, R. V. Ham, M. V. Damme, D. Lefeber, B. M. Y. Duran, and P. Beyl, "Exploiting natural dynamics to reduce energy consumption by controlling the compliance of soft actuators," Int. J. Robotics Research, vol. 25, no. 4, pp. 343-358, 2006.

33. M. Grebenstein and P. v. d. Smagt, "Antagonism for a highly anthropomorphic handarm system," Advanced Robotics, vol. 22, p. 3955, 2008.

34. S. Wolf and G. Hirzinger, "A new variable stiffness design: Matching requirements of the next robot generation," in IEEE Int. Conf. on Robotics and Automation. Pasadena, USA: IEEE, 2008, pp. 1741-1746.

35. H. Kobayashi and R. Ozawa, "Adaptive neural network control of tendon-driven mechanisms with elastic tendons," Automatica, vol. 39, pp. 1509-1519, 2003.

36. K. Tahara, Z.-W. Luo, R. Ozawa, J.-H. Bae, and S. Arimoto, "Bio-mimetic study on pinching motions of a dual-finger model with synergistic actuation of antagonist muscles," in IEEE International Conference on Robotics and Automation, 2006, pp. 994-999.

37. T. Wimböck, Ch. Ott, A. Albu-Schäffer, A. Kugi, and G. Hirzinger, "Impedance control for variable stiffness mechanisms with nonlinear joint coupling," in IEEE Int. Conf. on Intelligent Robotic Systems, 2008, pp. 3796-3803.

38. L. L. Tien, A. Albu-Schäffer, and G. Hirzinger, "Mimo state feedback controller for a flexible joint robot with strong joint coupling," Int. Conf. on Robotics and Automation (ICRA), 2007.

39. M. Weis, "Optimalsteuerung eines Robotergelenks mit einstellbarer passiver Steifigkeit," DLR and TU Karlsruhe Master Thesis, 2009.

40. S. Haddadin, T. Laue, U. Frese, S. Wolf, A. Albu-Schäffer, and G. Hirzinger, "Kick it like a safe robot: Requirements for 2050," Robotics and Autonomous Systems: Special Issue on Humanoid Soccer Robots, vol. 57, pp. 761-775, 2009. 